\section{MS21-P11 Structural disorder in a magnetic pyrochlore oxide}

Romain Sibille ${ }^{1,2}$, Tom Fennell ${ }^{2}$, Monica Ciomaga Hatnean ${ }^{3}$, Elsa Lhotel $^{4}$, David Keen ${ }^{5}$, Geetha Balakrishnan ${ }^{3}$, Michel Kenzelmann ${ }^{1}$

1. Laboratory for Scientific Developments and Novel Materials, Paul Scherrer Institut, 5232 Villigen, Switzerland

2. Laboratory for Neutron Scattering and Imaging, Paul Scherrer Institut, 5232 Villigen, Switzerland

3. Physics Department, University of Warwick, Coventry, CV4 7AL, UK

4. Institut Néel, CNRS and Université Joseph Fourier, BP 166 , 38042 Grenoble Cedex 9, France

5. ISIS Neutron Facility, STFC Rutherford Appleton Laboratory, Chilton, Didcot, OX11 0QX, UK

email: romain.sibille@psi.ch

Insulating pyrochlore oxides of formula $\mathrm{A}_{2} \mathrm{~B}_{2} \mathrm{O}_{7}$ where $\mathrm{A}$ is a rare-earth magnetic ion and $\mathrm{B}$ is a transition metal ion or a group IV ion, have attracted much attention because both A and B form a network of corner-sharing tetrahedra, a wonderful landscape for the study of magnetic frustration in three dimensions [1]. Rare-earth ions having a strong uniaxial anisotropy constrained along the $<111\rangle$ direction of the cubic unit-cell $\left(\mathrm{Pr}^{3+}\right.$, $\mathrm{Tb}^{3+}, \mathrm{Dy}^{3+}, \mathrm{Ho}^{3+}$ ) reverse, in theory, the role of ferromagnetic and antiferromagnetic couplings with regard to frustration [2]: the ferromagnetic case becomes highly frustrated, and can lead to the spin ice state [3], while the antiferromagnetic one is not. However, pyrochlore Ising antiferromagnets have proved able to markedly defy the expectations of long-range magnetic order. A famous example is the enigmatic terbium titanate, $\mathrm{Tb}_{2} \mathrm{Ti}_{2} \mathrm{O}_{7}$, which forms a so-called spin liquid state [4] with power-law spin correlations [5]. We report experimental studies on a related pyrochlore, $\mathrm{Tb}_{2} \mathrm{Hf}_{2} \mathrm{O}_{7}$, which displays striking similarities with the magnetism of $\mathrm{Tb}_{2} \mathrm{Ti}_{2} \mathrm{O}_{2}$ despite the existence of a substantial amount of structural disorder.

Firstly we present a detailed investigation of the crystal chemistry of $\mathrm{Tb}_{2} \mathrm{Hf}_{2} \mathrm{O}_{2}$ using a combination of Resonant Contrast Diffraction (RCD) and high-resolution Neutron Powder Diffraction (NPD), both on powder samples. We show that these measurements unveil the existence of defects that affect the pyrochlore structure of $\mathrm{Tb}_{2} \mathrm{Hf}_{2} \mathrm{O}_{7}$. The neutron Pair Distribution Function (PDF) presents significant differences between the experimental function at low $\mathbf{r}$ values and the one calculated from the average structure (Figure 1), which points out local distortions around the defects. Secondly we demonstrate that the structural defects in this material influence the magnetic ground state, and that our experimental results can be related to theories on disordered pyrochlore magnets.

[1] J. S. Gardner et al., Rev. Mod. Phys. 82, 53 (2010). [2] S. T. Bramwell et al., J. Phys. Condens. Matter 10, L215-220 (2012). [3] S. T. Bramwell and M. J. P. Gingras, Science 294, 1495 (2001). [4] J. S. Gardner et al., Phys. Rev. Lett. 82, 1012 (1999). [5] T. Fennell et al., Phys. Rev. Lett. 109, 017201 (2012).

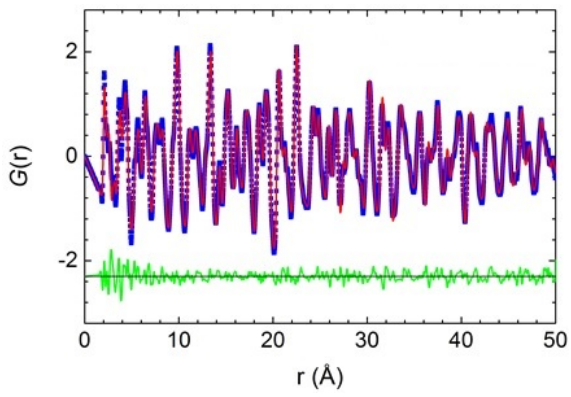

Figure 1. Pair Distribution Function obtained from neutron scattering measurements on GEM at ISIS.

Keywords: structural disorder, frustrated magnetism 